\title{
The TRAF2 and TRAF6 expression in myomas and myometrium of women in reproduction and perimenopausal age
}

\author{
Andrzej Plewka ${ }^{1}$, Pawel Madej ${ }^{2}$, Danuta Plewka ${ }^{3}$, Grażyna Nowaczyk ${ }^{3}$, Michał Morek ${ }^{1}$, \\ Edyta Bogunia $^{1}$, Monika Ciupińska-Kajor ${ }^{4}$, Karolina Sieroń-Stołtny ${ }^{5}$
}

\author{
${ }^{1}$ Department of Proteomics, Medical University of Silesia, Sosnowiec, Poland \\ ${ }^{2}$ Chair and Department of Gynaecological Endocrinology, Medical University of Silesia, Katowice, \\ Poland \\ ${ }^{3}$ Department of Histology, Medical University of Silesia, Katowice, Poland \\ ${ }^{4}$ Department of Histopathology, Medical University of Silesia, Katowice, Poland \\ ${ }^{5}$ Department of Internal Medicine, Medical University of Silesia, Bytom, Poland
}

\begin{abstract}
Uterine myomas represent one of the most common female diseases. Uterine myomas or fibromas are benign, hormone-responding tumours of, respectively, smooth muscles and fibroblasts and their aetiology induces a significant interest. In myomas the presence of aromatase was detected and, in addition, oestrogen was found to be synthesized in myoma cells. The studies were performed on myoma patients of generative age and those in peri-menopausal age. Expression of TRAF2 and TRAF6 proteins was examined using immunohistochemistry and Western blot approach in small and large uterine myomas isolated from women of various age. In addition, the evaluation was conducted at the periphery of every myoma. We indicated that the level of both tested proteins in myomas is higher than in control. TRAF2 level in myometrium was lower than in myomas but higher than in control. In the case of TRAF6 those changes were ambiguous. Age didn't have influence the level of expression in both tested TRAF in studied structures.
\end{abstract}

Key words: TRAF2, TRAF6, leiomyomas, small and large leiomyoma, women

\section{Introduction}

Despite the high frequency of leiomyoma manifestation scanty information is available on their development and growth. On the basis of biochemical and cytogenetic studies every leiomyoma is thought to represent a product of clonal expansion of an individual myocyte [1-3]. Just as it is the case with other tumours, probably several genetically sensitive sites exist, which lead to dysregulation of a smooth muscle cell, resulting in its transformation to the common phenotype of a leiomyoma cell.

Studies performed till now demonstrated that an immune response is linked to activation of NF- $\mathrm{KB}$

Correspondence: A. Plewka, Department of Proteomics, Medical University of Silesia, ul. Ostrogórska 30, 41-200 Sosnowiec, Poland tel.: (+4832) 3641430, fax.: (+4832) 3641440, e-mail: aplewka@sum.edu.pl under effect of IL-1 and TNF- $\alpha$ [4-8]. The two cytokines exert their effect due to activation of

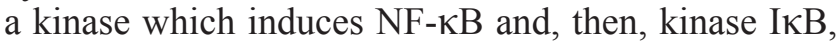
which directly affects activity of NF- $\kappa \mathrm{B}$ transcription factor. The phosphorylated, C-terminal domain of IL-1 receptor-linked kinase interacts with TRAF6 protein [9-11]. Transmission of IL-1 signal may include role of TRAF6 and/or TRAF2 [12-14]. TRAF-6 activates NIK kinase but it should be stressed that NIK is a common mediator of signalling pathways originating from multiple cytokine receptors [15-17].

TRAF (TNF receptor-associated factor) family represents a family of cytoplasmic proteins capable both of negative control of apoptosis and of induction of genes which promote survival. They were shown to serve as adaptor proteins for a broad range of surface receptors, to play an important role not only in apoptosis but also in response to stress. The proteins may directly interact with intracellular domains of surface 
receptors, including TNF- $\alpha$ (TRAF2) or IL-1 $\beta$ (TRAF6) receptors.

TRAF proteins may serve for modulation of receptors capacities to mobilize various signalling pathways, which lead to phosphorylation and activation of protein kinases and, then, to activation of transcription factors of Rel and AP-1 family.

In our earlier study we evaluated expression of aromatase in uterine myomas [18]. Expression of the aromatase depends on several factors, including TRAF. Even if neither TRAF2 nor TRAF6 directly affect expression of aromatase, their indirect effect is evident. The two proteins are known to directly stimulate activity of NF-KB. This nuclear factor may bind one of the promoters, such as I.4, which mobilizes aromatase synthesis pathway. Expression of aromatase may also be stimulated by PII promoter. While the promoter is activated by $\mathrm{PGE}_{2}$, levels of the prostaglandin are is directly related to activity of COX-2, and activity of the cyclooxygenase is stimulated, among others, by NF- $\mathrm{\kappa B}$.

In this aim the attempt will be undertaken to demonstrate whether there exist differences in the amount and distribution of TRAF2 and TRAF6 proteins in myomas of various size, isolated from women of various age, as compared to an unchanged tissue. The evaluation will be made using Western blot technique and quantitative analysis of immunohistochemical tests conducted on tissues of a normal uterus and a myomatous uterus.

\section{Materials and methods}

Human material. Recruitment of patients, clinical studies and hormonal tests will be conducted in the Chair and Department of Gynaecological Endocrinology, Medical University of Silesia in Katowice while enzymatic and protein studies will be executed in the Department of Proteomics.

The studies were conducted on 40 patients with myomas, at the reproductive age (below $45^{\text {th }}$ year of age, $\mathrm{FSH}<30 \mathrm{mIU} / \mathrm{ml}$; Samales were taken in follicular phase of menstrual cycle) and 40 patients with myomas in the perimenopausal age (45-55 years of life, FSH>30 mIU/ml). Inclusion criteria will involve myoma detected by USG, qualification of the patient to hysterectomy, informed consent to the planned studies. The exclusion criteria will include: therapy with any drugs, including hormonal drugs in the minimum of 3 months before inclusion to the studies, neoplastic disease, endometrial hypertrophy, metabolic and systemic disturbances, nicotinism.

Myometrial samples, for use as health controls were taken from 10 young women $(<40$ years old $)$ undergoing hysterectomies for ovary tumors and 10 perimenopausal age women $(>52$ years old) undergoing hysterectomies for uterine prolapse.

In these studies we used only material from uteruses with one large myomas or one large and few small myomas. The material for studies was excised from uterus taken during surgery, and fixed histopathologically. Samples of leyomyoma $1 \times 1 \mathrm{~cm}$ and samples of a similar size from health myometrium at the distance of at least $4 \mathrm{~cm}$ each were taken. Before isolation of myometrium samples the patients were informed on the aim of the studies. The planned investigative procedures were approved by the Medical Bioethical Commission.

\section{Characteristics of studied groups}

Group 1. Myometrium of menstruating women, in whom hysterectomy was performed for reasons other than uterine leiomyomas; the 10 patients represented the lesion-free control.

Group 2. Leiomyomas of $<3 \mathrm{~cm}$ in diameter, sampled from uteri of menstruating women ( $\mathrm{n}=20$ women).

Group 3. Myometrium of menstruating women, sampled at the distance of not less than $4 \mathrm{~cm}$ from margins of a small leiomyoma ( $\mathrm{n}=20$ women; autologous control).

Group 4. Leiomyomas of $>5 \mathrm{~cm}$ in diameter, sampled from uteri of menstruating women ( $\mathrm{n}=20$ women).

Group 5. Myometrium of menstruating women, sampled at the distance of not less than $4 \mathrm{~cm}$ from margins of a large leiomyoma ( $\mathrm{n}=20$ women; autologous control).

Group 6. Myometrium of non-menstruating women, in whom hysterectomy was performed for reasons different than uterine leiomyomas ( $\mathrm{n}=10$ women; lesion-free control).

Group 7. Leiomyomas of $<3 \mathrm{~cm}$ in diameter, sampled from uteri of non-menstruating women ( $\mathrm{n}=20$ women).

Group 8. Myometrium of non-menstruating women, sampled at the distance of not less than $4 \mathrm{~cm}$ from margins of a small leiomyoma ( $\mathrm{n}=20$; autologous control).

Group 9. Leiomyomas of $>5 \mathrm{~cm}$ in diameter, sampled from uteri of non-menstruating women, $(n=20$ women $)$.

Group 10. Myometrium of non-menstruating women, sampled at the distance of not less than $4 \mathrm{~cm}$ from margins of a large leiomyoma ( $\mathrm{n}=20$ women, autologous control).

Immunohistochemistry. Tissues were fixed in $4 \%$ buffered formalin, dehydrated and then embedded in paraffin. Section $(5 \mu \mathrm{m})$ were mounted on silane-coated slides. Samples were de-waxed, and rehydrated. To unmask the antigen, sections were boiled in $0,01 \mathrm{M}$ citrate buffer, $\mathrm{pH}$ 6, in a microwave oven for $10 \mathrm{~min}$ at 800 W. Endogenous peroxidase activity was quenched using $1.5 \%(\mathrm{v} / \mathrm{v})$ solution $\mathrm{H}_{2} \mathrm{O}_{2}$ in methanol for $10 \mathrm{~min}$ and then washed in PBSTween $20(0,05 \% \mathrm{v} / \mathrm{v})$ and blocked with $1 \%$ BSA for $60 \mathrm{~min}$. The sections were further blocked with avidin-biotin-blocking solution according to the manufacturer's instruction. After this the slides were incubated with rabbit anti-TRAF2 polyclonal antibody (Abcam) or rabbit anti-TRAF6 monoclonal antibody (Abcam) in a humidified chamber for $22 \mathrm{~h}$ at $4^{\circ} \mathrm{C}$. After washing in PBSTween 20 sections were incubated with biotinylated goat anti-rabbit immunoglobulins (Vector Laboratories Inc.) for $30 \mathrm{~min}$, and were incubated with avidin-biotinylated peroxidase complex (Vector) for $30 \mathrm{~min}$. The bound antibodies were visualised with diaminobenzidine (DAB) and $\mathrm{H}_{2} \mathrm{O}_{2}$ in PBS, $\mathrm{pH} 7,5$ according to supplier's instructions (Vector). Finally, the tissues were stained with Gill's hematoxilin, dehydrated, and cover-slipped. For negative controls rabbit IgG were used.

In each positively stained cell, the intensity of staining was measured as the optical density of the reaction product, with the program KS 300 VIDAS video image analyzer served by IBAS 2.5 system and a Panasonic digital camera. For each analyzed area, $173 \times 130 \mu \mathrm{m}$ average optical density per unit area was calculated. Finally, the arithmetic mean and standard deviation were calculated.

Western blotting. Tissue samples $(\sim 500 \mathrm{mg})$ were homogenized in $0.5 \%$ Triton X-100 lysis buffer containing $20 \mathrm{mM}$ Tris$\mathrm{HCl}(\mathrm{pH} 7.5), 150 \mathrm{mM} \mathrm{NaCl}, 10 \mathrm{mM} \mathrm{NaF}, 2 \mathrm{mM}$ dithiothreitol (DTT), 1mM sodium orthovanadate, $1 \mathrm{mM}$ phenylmethylsulfonyl floride (PMSF) and $20 \mathrm{M}$ aprotinin. Homogenates were centrifuged at $10,000 \times \mathrm{g}$ for $10 \mathrm{~min}$. Concentration of protein was determined by Bradford assay. For immunoblotting samples (25 $\mu \mathrm{g}$ of protein) were separated by $12 \%$ SDS-polyacrylamide gels, and transferred to nitrocellulose membranes. After blocking with $5 \%$ skim milk in PBS-T (PBS with $0.1 \%$ Tween 20 ) for $1 \mathrm{~h}$, the membranes were probed with TRAF2 or TRAF6 antibodies (Abcam) overnight. Primary antibodies were detected with 
biotinylated anti-rabbit IgG made in goat (Vectastain ABC-AmP Vector Laboratories, Inc.) (30 min) and with streptavidin and biotinylated alkaline phospatase complex (30 min) and developed using a nitroblue tetrazolium and 5-bromo-4-3-indolylphosphate (Vector Laboratories, Inc.). Subsequent Western blotting analyses were performed.

Statistical analysis. Normal distribution of the data was confirmed by the Kolmogorov-Smirnov test. Results are presented as a mean \pm SD. The Student's t-test or the Mann-Whitney U-test were performed where appropriate. To estimate correlation between TRAFs expression, myomas and age of women we used Spearman's rank correlation test. A p value $<0.05$ was considered statistically significant.

\section{Results}

\section{Immunohistochemical studies}

Optical density of cells with expression of an evaluated protein reflects concentration of the immunocytochemical reaction product in evaluated uterine structures.

\section{TRAF2}

\section{Young women}

Evaluating levels of TRAF2 in studied structures (Fig. 1), optical density of the immucytochemical reaction products in small myomas of young women was found to be higher, amounting to around $185 \%$ of the control level (Fig. 2). The same analysis targeted at vicinity of the myomas demonstrated also higher expression of the protein, which in this case reached $152 \%$ of the control level. Analysis of the data showed that expression of TRAF2 at the periphery of myomas amounted to $82 \%$ of the value noted in the myoma.

Comparing optical density in large myomas manifesting TRAF2 expression with the control level the demonstrated expression of the protein was found to be equal to $196 \%$ of the control level. Similarly to small myomas, TRAF2 level measured at the periphery was slightly lower than that in the myomas, amounting to $182 \%$ of the control level. This corresponded to $93 \%$ of TRAF2 expression level noted in large myomas.

In women of reproductive age, TRAF2 expression level in large uterine myomas resembled the value noted in small myomas. Evaluation of the protein expression at the direct periphery of the large myomas demonstrated a similar expression level to that noted at the periphery of small myomas.

\section{Women at perimenopausal age}

Evaluation of TRAF2 level in studied structures (Fig. 1) showed that in women of perimenopausal age optical density of the reaction product in small myomas was increased to almost $165 \%$ of the control level (Fig. 2). Analysis of myoma periphery demonstrated the level higher than in the control $(150 \%$ of the control level). Analysis of the data disclosed that expression of TRAF2 at the periphery of small myomas constituted $92 \%$ of the value observed inside the myomas.

Evaluating optical density in large myomas with TRAF2 expression as compared to the control, the former was found to manifest higher expression of the protein, amounting to almost $175 \%$ of the control level. In contrast to small myomas, activity of TRAF2 measured at the periphery was lower than inside the myoma and amounted to around $147 \%$ of the control level.

Analysis of TRAF2 expression in uterine myomas of women of post-reproductive age disclosed that in large myomas expression of TRAF2 was comparable to the expression documented in small myomas. At the direct vicinity of large myomas the expression resembled that observed around small myomas.

\section{Effect of age}

Evaluating content of TRAF2 reaction product, a level of the protein in control samples obtained from young women was slightly lower than in women of perimenopausal age women. A similar observation was made upon quantitation of the protein expression in small myomas and at their periphery in women of the two age groups.

Analysis of the quantitative data related to large myomas demonstrated that in women of perimenopausal age TRAF2 expression level was lower and amounted to $89 \%$ of the level detected in young women. Also at the periphery of large myomas the level was distinct in the two age groups: in young women it amounted to $124 \%$ of the value observed in the older group.

\section{TRAF6 \\ Young women}

Upon evaluation of TRAF6 levels in the studied structures (Fig. 3), in young women optical density of the immunocytochemical reaction product was increased in small myomas, reaching around $235 \%$ of the control level (Fig. 4). The same analysis targeted at the myoma periphery also in this case demonstrated expression of the protein higher than in the control, reaching the level of $185 \%$. Analysis of the data demonstrated that expression of TRAF6 at the periphery of myoma amounted to $78 \%$ of the value noted in the myoma.

Comparing optical density of large myomas with TRAF6 expression with the control level, the protein expression was found to represent $288 \%$ of the control value. Similarly to small myomas, TRAF6 expression level measured at the periphery was slightly lower than that in myomas and corresponded to $166 \%$ of the con- 
trol level or $58 \%$ of the expression of TRAF 6 noted in large myomas.

Quantitative analysis of TRAF6 expression level in uterine myomas of women in reproductive age, in large myomas the expression level was higher than that observed in small myomas, amounting to $123 \%$ of the protein expression level in small myomas. In direct vicinity of large myomas the expression level was lower than the level noted around small myomas, corresponding to $90 \%$ of the latter level.

\section{Women of perimenopausal age}

Evaluation of TRAF6 expression level in the studied structures (Fig. 3) disclosed that in women of perimenopausal age optical density of the reaction product increased in small myomas to almost $210 \%$ of the control level (Fig. 4). At periphery of the myomas expression of the protein also was higher than in the control, reaching the level equivalent to $195 \%$ of the control. Analysis of the data showed that TRAF6 expression at periphery of small myomas reached $95 \%$ of the value observed in the myomas.

Evaluation of optical density of large myomas with TRAF6 expression showed that the expression was higher, corresponding to $240 \%$ of the control level. Similarly to small myomas TRAF6 activity measured at periphery was slightly lower than that in the myomas, amounting to around $222 \%$ of the control value.

Analysis of TRAF6 expression in uterine myomas of women in post-reproductive age demonstrated that in large myomas TRAF6 expression was higher as compared to the expression observed in small myomas. In a direct vicinity of large myomas it was found to be slightly higher than the level noted in vicinity of small myomas.

\section{Effect of age}

Comparison of the product content in the reaction for TRAF6 showed levels of the protein in control samples originating from young women was slightly higher than the level observed in perimenopausal women. A similar observation was made during quantitative evaluation of the protein expression in small myomas. Periphery of small myomas demonstrated similar values of TRAF6 expression in the two age groups of women.

Analysis the quantitative data related to large myomas demonstrated that TRAF6 expresssion level in women of perimenopausal age was lower, amounting to $83 \%$ of the level demonstrated in young women. The reaction product measured in vicinity of large myomas was also distinct in the two age groups: in young women it amounted to $75 \%$ of the value observed in the older group.

\section{Western blot analysis TRAF2}

Upon comparison of TRAF2 expression in myometrium of women of various age the samples originating from women in perimenopausal age were found to be slightly higher, although the difference failed to reach statistical significance.

For a better and a more legible evaluation of changes in TRAF2 expression (and the remaining proteins evaluated by Western blot analysis) a quantitative analysis was performed exclusively in the same age groups, in every case accepting the expression value noted in a pure control as $100 \%$.

Expression of TRAF2 measured at the periphery of myomas originating from uteri of young females was higher than that in the control, amounting to $115 \%$ and $120 \%$ (Fig. 5; Table 1) in, respectively small and large myomas although the differences proved insignificant.

The TRAF2 expression level detected in small myomas isolated from uteri of young women was clearly higher than in the control and amounted to $135 \%$, and the difference was significant. Even higher was TRAF2 expression level detected in large myomas, in which the expression level reached $140 \%$ of the control level.

Upon analysis of differences in TRAF2 expression between myomas and their direct vicinity expression of the protein in small myomas was significantlly higher than that in the surrounding myometrium. A similar difference in the level of TRAF2 expression was detected in cases of large myomas.

Analysis of differences in TRAF2 expression in uteri of perimenopausal women demonstrated similar but slightly more accentuated differences: expression of the protein measured at the periphery of myomas was also higher than that in the control. The levels amounted to $125 \%$ and $120 \%$ in small and large myomas, respectively (Fig. 5; Table 1), but also in this case the differences proved to be insignificant.

Expression level of the evaluated TRAF2 protein in small myomas isolated from uteri of perimenopausal women was higher than that in the control and reached $145 \%$, and the difference was significant. In large myomas of the age group TRAF2 expression level was even higher, amounting to $160 \%$ of the control level.

Evaluating TRAF2 expression level in myomas and in the surrounding myometrium it was shown that in the older age group expression of TRAF2 in small myomas was significantly higher than that in the surrounding myometrium. An even higher difference, of $135 \%$ order was disclosed during analysis of differences in TRAF-2 expression levels in large myomas. 

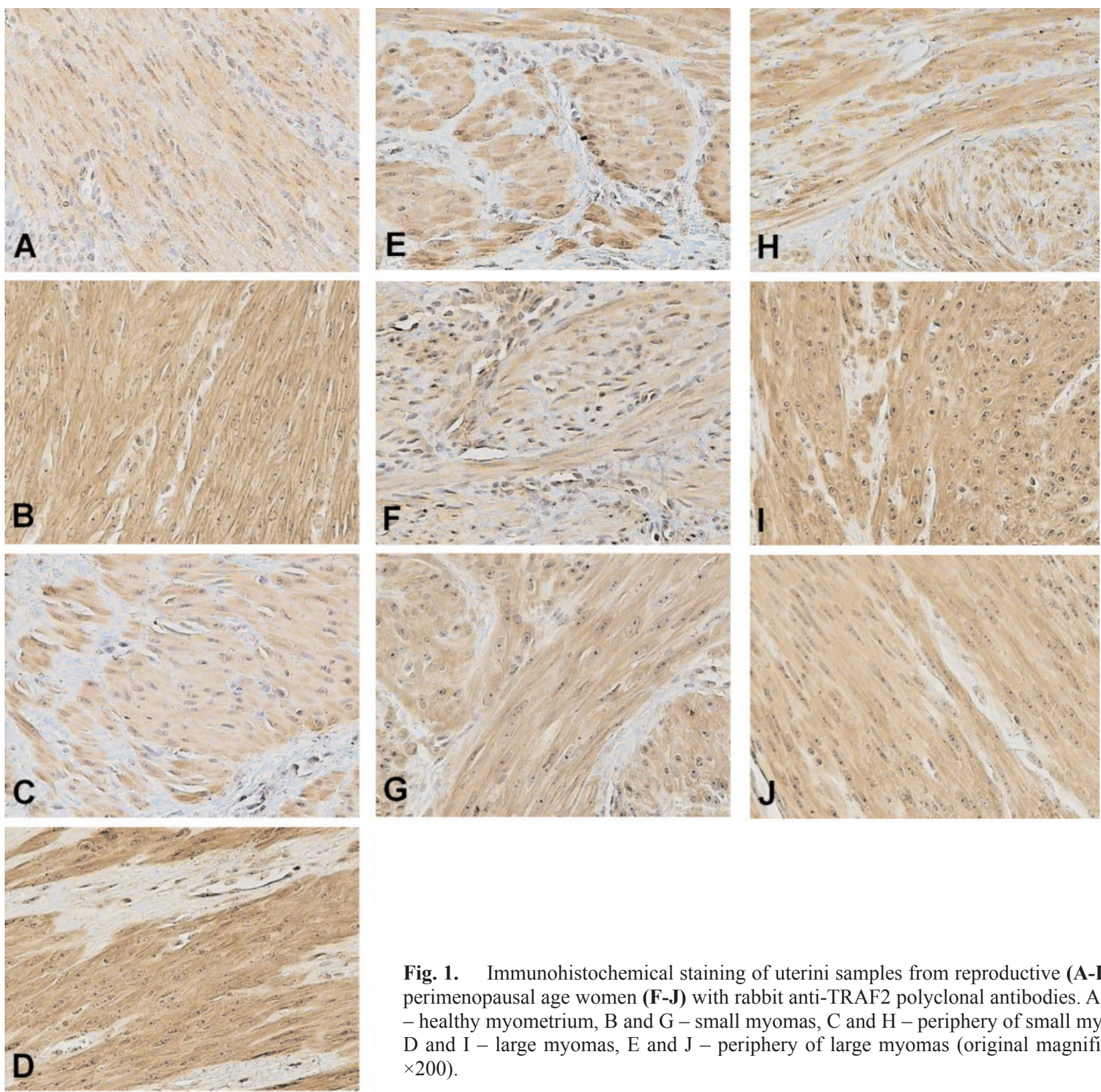

Fig. 1. Immunohistochemical staining of uterini samples from reproductive (A-E) and perimenopausal age women (F-J) with rabbit anti-TRAF2 polyclonal antibodies. A and $\mathrm{F}$ - healthy myometrium, $\mathrm{B}$ and $\mathrm{G}$ - small myomas, $\mathrm{C}$ and $\mathrm{H}$ - periphery of small myomas, $\mathrm{D}$ and $\mathrm{I}$ - large myomas, $\mathrm{E}$ and $\mathrm{J}$ - periphery of large myomas (original magnification $\times 200)$.

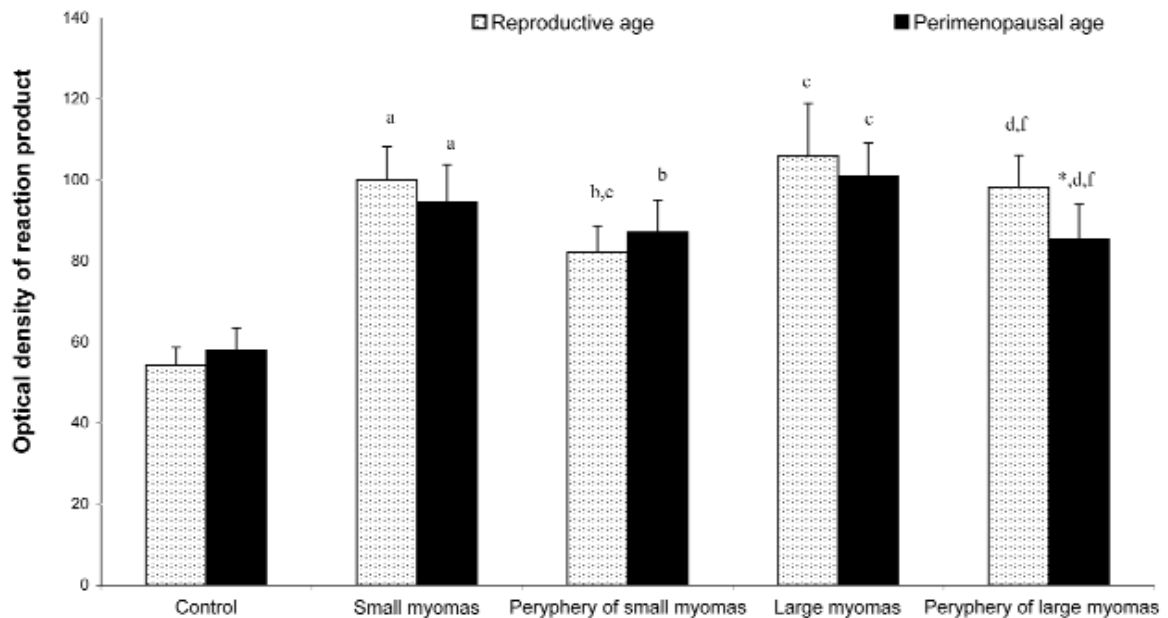

Fig. 2. Quantitative evaluation of TRAF2 expression in immunohistochemical staining. Date shown represent mean $\pm \mathrm{SD}$.

Differences in TRAF2 expression: ${ }^{*}-$ in reproductive age women and perimenopausal age women; $\mathbf{a}$ - in small myomas and healthy myometrium; $\mathbf{b}-$ in periphery of small myomas and healthy myometrium; c - in large myomas and healthy myometrium; $\mathbf{d}$ in periphery of large myomas and healthy myometrium; e - in small myomas and its periphery; $\mathbf{f}$ - in small myomas and its periphery, are statistically significant. 

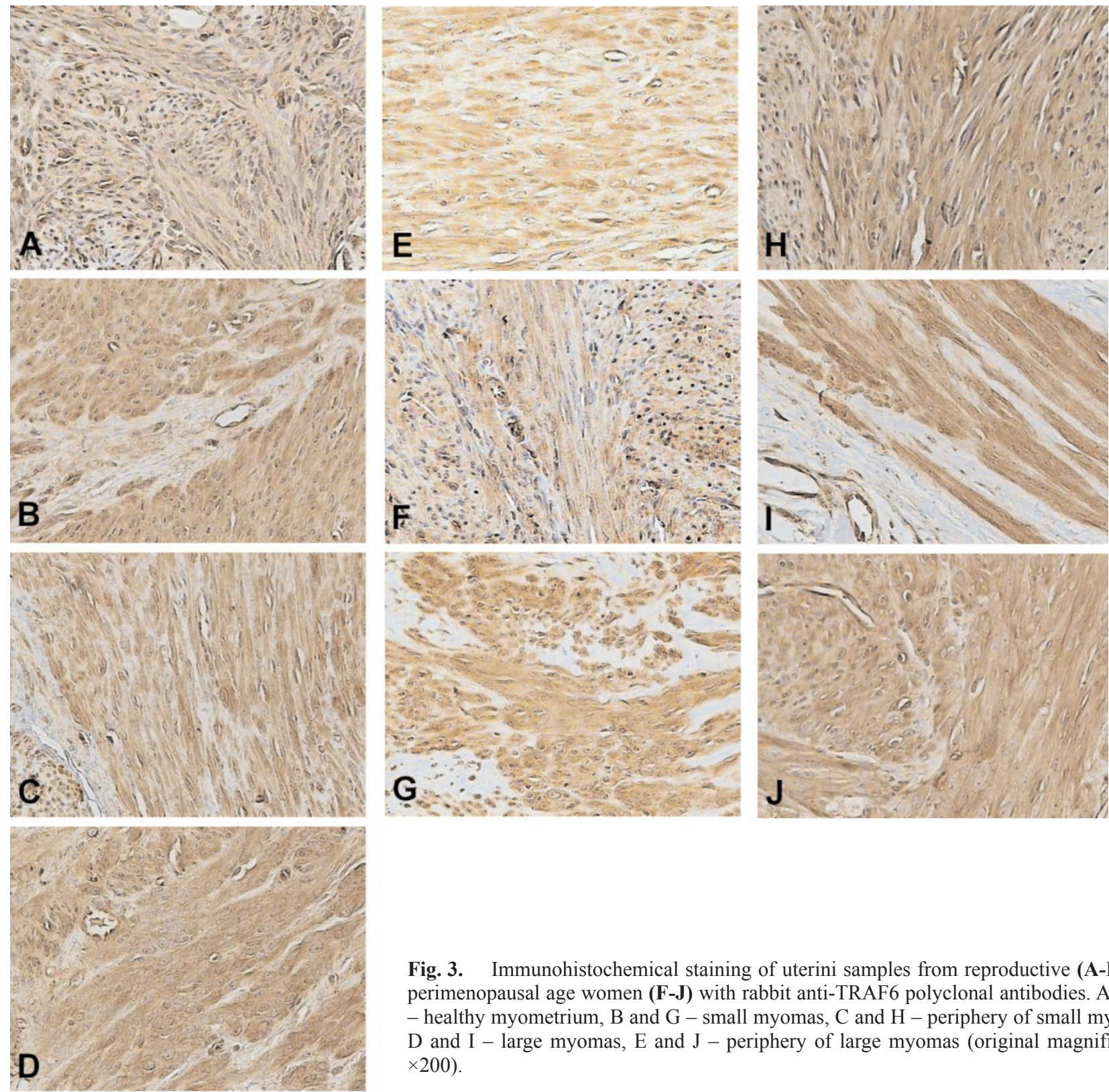

Fig. 3. Immunohistochemical staining of uterini samples from reproductive (A-E) and perimenopausal age women (F-J) with rabbit anti-TRAF6 polyclonal antibodies. A and F - healthy myometrium, B and $\mathrm{G}$ - small myomas, $\mathrm{C}$ and $\mathrm{H}$ - periphery of small myomas, $\mathrm{D}$ and $\mathrm{I}$ - large myomas, $\mathrm{E}$ and $\mathrm{J}$ - periphery of large myomas (original magnification $\times 200$ ).

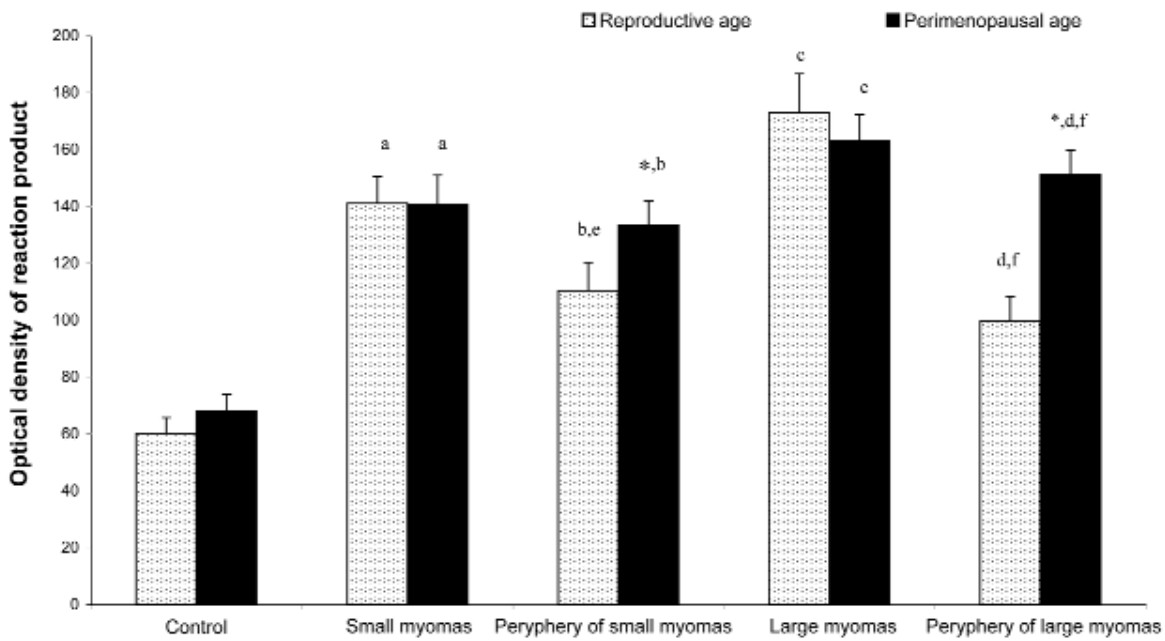

Fig. 4. Quantitative evaluation of TRAF6 expression in immunohistochemical staining. Date shown represent mean \pm SD. Differences in TRAF6 expression: ${ }^{*}$ - in reproductive age women and perimenopausal age women; a - in small myomas and healthy myometrium; $\mathbf{b}$ - in periphery of small myomas and healthy myometrium; $\mathbf{c}$ - in large myomas and healthy myometrium; $\mathbf{d}$ - in periphery of large myomas and healthy myometrium; e - in small myomas and its periphery; $\mathbf{f}$ - in small myomas and its periphery, are statistically significant. 
Table 1. Western blot analysis of TRAF2 protein in uterus tissue samples from myometrium and leiomyomas from young and perimenopausal age women. Quantitative analysis indicated, that TRAF2 level in myometrium perimenopausal age women is $107 \%$ those in young women.

\begin{tabular}{|l|c|c|c|c|}
\hline \multirow{2}{*}{ Control } & \multicolumn{2}{|c|}{ Small myomas } & \multicolumn{2}{c|}{ Large myomas } \\
\cline { 2 - 5 } & Myomas & Miometrium & Myomas & Miometrium \\
\hline Reproductive age women - 100 & $135 \pm 7^{\mathrm{a}}$ & $115 \pm 4^{\mathrm{e}}$ & $140 \pm 8^{\mathrm{c}}$ & $120 \pm 4^{\mathrm{d}, \mathrm{f}}$ \\
\hline Perimenopausal women - 100 & $145 \pm 4^{\mathrm{a}}$ & $125 \pm 7^{\mathrm{b}, \mathrm{e}}$ & $160 \pm 8^{* \mathrm{c}}$ & $120 \pm 5^{\mathrm{f}}$ \\
\hline
\end{tabular}

Differences in TRAF2 expression: ${ }^{*}$ - in reproductive age women and perimenopausal age women; ${ }^{a}$ in small myomas and healthy myometrium; ${ }^{b}$ in periphery of small myomas and healthy myometrium; ${ }^{\mathrm{c}}$ in large myomas and healthy myometrium; ${ }^{\mathrm{d}}$ in periphery of large myomas and healthy myometri$\mathrm{um} ;{ }^{\mathrm{e}}$ in small myomas and its periphery, ${ }^{\mathrm{f}}$ in large myomas and its periphery, are statistically significant for $\mathrm{p}=0.05$.

Table 2. Western blot analysis of TRAF6 protein in uterus tissue samples from myometrium and leiomyomas from young and perimenopausal age women. Quantitative analysis indicated, that TRAF6 level in myometrium perimenopausal age women is $113 \%$ those in young women.

\begin{tabular}{|c|c|c|c|c|}
\hline \multirow{2}{*}{ Control } & \multicolumn{2}{|c|}{ Small myomas } & \multicolumn{2}{|c|}{ Large myomas } \\
\hline & Myomas & Miometrium & Myomas & Miometrium \\
\hline Reproductive age women - 100 & $125 \pm 6^{\mathrm{a}}$ & $110 \pm 5^{\mathrm{e}}$ & $135 \pm 4^{\mathrm{c}}$ & $110 \pm 4^{f}$ \\
\hline Perimenopausal women - 100 & $135 \pm 6^{\mathrm{a}}$ & $135 \pm 7^{\mathrm{b}}$ & $145 \pm 5^{* \mathrm{c}}$ & $125 \pm 5^{\mathrm{d}, \mathrm{f}}$ \\
\hline
\end{tabular}

Differences in TRAF6 expression: ${ }^{*}$ - in reproductive age women and perimenopausal age women; ${ }^{a}$ in small myomas and healthy myometrium; ${ }^{\mathrm{b}}$ in periphery of small myomas and healthy myometrium; ${ }^{\mathrm{c}}$ in large myomas and healthy myometrium; ${ }^{\mathrm{d}}$ in periphery of large myomas and healthy myometri$\mathrm{um}^{\mathrm{e}}{ }^{\mathrm{e}}$ in small myomas and its periphery, ${ }^{\mathrm{f}}$ in large myomas and its periphery, are statistically significant for $\mathrm{p}=0.05$.

\section{TRAF6}

Comparison of TRAF6 expression levels in myometrium of women of various age groups showed that in samples originating from perimenopausal women it was even higher, reaching $120 \%$ of the value noted in the younger group. However, the difference proved to be insignificant.

Expression of TRAF6 measured at the periphery of myomas originating from uteri of young women resembled that in the control, amounting to, at most, $110 \%$ of the control value.

TRAF6 expression level in small myomas isolated from uteri of young women was higher than that in the control and amounted to $125 \%$, but the difference was insignificant. Even higher level of TRAF6 expression was seen in large myomas, in which the level of the discussed TRAF protein reached $135 \%$ of the control level (Fig.6; Table 1).

In analysis of TRAF6 levels in myomas and in the surrounding myometrium it was demonstrated that in the group of young women expression of TRAF6 in small myomas was slightly, but insignificantly higher than in the surrounding myometrium. A more pronounced difference, of $125 \%$ order, was detected during analysis differences in TRAF6 expression levels in large myomas, as compared to those in their direct neighbourhood.

Analysis of TRAF6 expression in uteri of perimenopausal women demonstrated more pronounced differences: expression of the protein measured at the periphery of myomas was higher than that in the control, amounting to $135 \%$ and $125 \%$ in small and large myomas, respectively (Fig. 6; Table 1). The difference was significant for periphery of small myomas.

The detected expression level of the evaluated TRAF6 protein in small myomas isolated from uteri of peri-menopausal women was higher than that in the control, amounting to $135 \%$, and the difference was significant. In large myomas of the age group the detected TRAF6 expression level was even slightly higher, reaching $145 \%$ of the control value.

Evaluation of TRAF6 expression level in myomas and the surrounding myometrium demonstrated that in the older age group expression of TRAF6 in small myomas was the same as in the surrounding myometrium. On the other hand, a difference of $115 \%$ order was noted upon analysis of TRAF6 expression in large myomas. However, the difference was insignificant.

\section{Discussion}

Uterine leiomyomas are the most frequent tumours of female genital tract. More than $70 \%$ of preparations, resulting from hysterectomy performed for various reasons, demonstrated presence of uterine leiomyomas [19-21].

Certain traits of TRAF proteins suggest that they act as cytoplasmic adapters, which may support transduction of an intra-cellular signal due to their capacity to bind receptors, including their self-recruitment to the signalling complex. It is known that TRAF pro- 


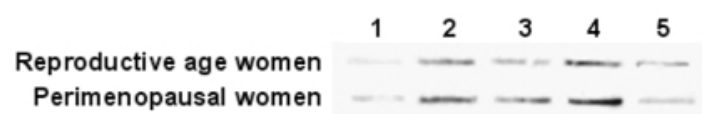

Fig. 5. There is Western blot analysis of TRAF2 in myometrium and leiomyoma tissue from reproductive age women and perimenopausal age women. Lane 1 - control; lane 2 - small myomas; lane 3- periphery of small myomas; lane 4 - large myomas; lane 5 - periphery of large myomas.Samples $(25 \mu \mathrm{g} /$ lane $)$ were electrophoresed, blotted and TRAF2was detected (see Materials and Methods and Table 1).

teins may serve in modulation of receptor's capacity to induce various signalling pathways [22], which lead to phosphorylation and activation of protein kinases and, then, to activate transcription factors of, among others, AP-1 family.

In our studies we have demonstrated that myometria of myoma-free women manifest evident expressions of TRAF2 and TRAF6 proteins. Their levels were well marked but similar in samples originating from young women and women of perimenopausal age (Fig. 2 and 4; Table 1 and 2). This indicates that absence of diagnosis of myoma presence not necessarily is equivocal with an absence of neoplastic risk in a patient. A stable level of TRAF2 and TRAF6 expression represents a stimulating factor in the NF- $\mathrm{KB}$ activation pathway, which, as generally known, directly affects proliferative processes. Even if neither TRAF2 nor TRAF6 affect directly expression of aromatase, their indirect effect is evident. As generally known, the two proteins directly stimulate activity of NF- $\mathrm{KB}$. This nuclear factor may associate with one of promoters, such as I.4, which mobilizes aromatase synthesis pathway.

TRAF proteins also seem to play a significant role in modulation of an early stage in the receptor-induced activation of NF-kB $[9,23,24]$. As has been demonstrated, NF- $\mathrm{KB}$-induced kinase (NIK) represents a part of the signalling complex, formed by multimerization of TRAF proteins following recruitment to cell surface receptors [15,25-27]. An evidence for the ability of TRAF proteins to affect activation of NF- $\mathrm{KB}$ with receptor mediation has originated from the demonstration that TRAF2 may inhibit activation of NF- $\mathrm{KB}$ in response to oligomerization of numerous TNFR-linked molecules, including TNFRII, CD40 and other [28]. In our studies we have shown that expression of TNF- $\alpha$ has been increasing in all studied experimental groups (unpublished data). The cytokine directly activates, among others, TRAF2. Subsequently we have shown that also IL-1 reached high levels, particularly in cases of small and large myomas (unpublished data). In concert with TNF- $\alpha$ the cytokine stimulates TRAF6 [29]. The two studied heretofore proteins exert a direct stimulatory effect on NF-kB activity. This may be synonymous to an increased proliferation in the pathologically

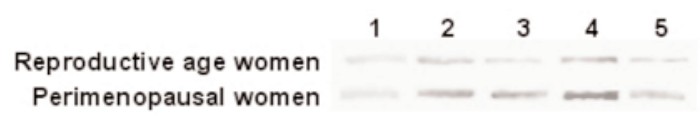

Fig. 6. There is Western blot analysis of TRAF6 in myometrium and leiomyoma tissue from reproductive age women and perimenopausal age women. Lane 1 - control; lane 2 - small myomas; lane 3- periphery of small myomas; lane 4 - large myomas; lane 5 - periphery of large myomas. Samples $(25 \mu \mathrm{g} /$ lane $)$ were electrophoresed, blotted and TRAF6 was detected (see Materials and methods and Table 2).

altered tissue. However, it is suggested that engagement of the proteins may activate NF- $\kappa B$ in more than a single pathway. Although certain TRAF, and TRAF2 in particular, activate NF-KB and AP-1, the differential recruitment of TRAF-activating or TRAF-inhibiting molecules seems to involve just one of several control levels in receptor-induced signal transduction. The fact that TRAF-binding sites in receptors significantly differ in sequence provides an argument for differences in affinity of TRAF molecules binding to various receptor proteins. Moreover, the potential for homo- and/or heterodimerization of TRAF molecules, which leads to formation of higher order complexes might add here another level of control [12]. It is probable that differences in levels of TRAF expression and/or depletion of various TRAF molecules following recruitment to the receptor and the nature of multimerized, TRAF-recruiting receptor result in formation of various poly-protein aggregates and these either allow cells to survive or induce apoptosis [28,30-32].

A few experiments provided the proof for action of TRAF proteins as mediators of life or death and represented subsequent steps in understanding the role of TRAF as one of the key controllers of cell reaction to stress. The clearly higher levels of TRAF2 and TRAF6 expression, documented in both types of myomas and at their periphery may corroborate the hypothesis. As described above, TRAF2 and TRAF6 seem to act as inducers of positive signals for cell growth and proliferation, with mediation of kinase cascades and induction of NF-kB-controlled genes [33]. When the molecules are available in significant quantities, activation of TNFR seems to promote stress response. In contrast, in cells in which the factors are depleted or blocked by inhibitors, the apoptotic response is favoured [28,30-32]. Thus, availability of TRAF proteins and their activity may regulate the key point of cell survival, involving on one hand stress response and, on the other, a programmed cell death.

The data provide proof for important role of TRAF2 in activation of JNK pathway through TNFR. Even if experiments in tissue culture systems indicate that TRAF2 may control activation of NF- $\mathrm{KB}$, existence of TRAF-independent pathways has been 
demonstrated. Evidently this might have been expected since, as we have shown, TRAF2 level in myomas was lower than the level of TRAF6.

The experiments suggest that TRAF2 may exert also an anti-apoptotic activity. Studies on cells with deficiency of TRAF2 confirmed the anti-apoptotic activity of TRAF2 and its significance for TNFinduced activation of JNK [34-36]. Thus, even if TRAF2 manifests an evident anti-apoptotic activity, it also can bind members of TNFR family to pro-apoptotic pathways.

Literature references related to the subject are quite scanty. In the few studies on local expression of TRAF2 and TRAF6 in uterine myomas an attempt was made to find out if proteins manifested in myoma cells play a significant role in their growth and if myomas synthesize sufficient amounts of oestrogens for promotion of myoma growth. Myoma cells are thought to be able to synthesize appropriate amounts of oestrogens and, moreover, the local aromatization in myoma tissue is thought to be responsible for the cell growth-promoting effect.

In our paper we were defining level of TRAF expression with two techniques. We indicated that profiles of quality changes are the same but quantity changes are different. One can notice that results received in immunohistochemical stains are visibly higher than the one received in Western blot method. Lower values obtained in Western blot method are is probably caused by the fact that staining in this method is the applied to whole mass of sample in the distinction of immunohistochemical staining where its apply exclusively to cells.

In discussions on TRAF proteins, an important observation should be taken into account: in our studies we have noted that in myometria originating from women with myoma presence, expression of TRAF proteins has been evident. Its level has been even higher than that in myomas and clearly higher than the level seen in a healthy myometrium. It should be borne in mind that the samples were isolated outside of the so called surgical safety margin and, thus, they originated from the region of uterus, in which no relapse of myoma should occur. In the region our studies have documented TRAF protein expression sufficiently high to warrant in appropriate conditions the development of de novo myoma. It remains to be established how far from the tumorous lesion the level of TRAF protein expression is sufficient for such a complication. This requires further studies.

\section{References}

[ 1] Pandis N, Heim S, Bardi G, Flodérus UM, Willén H, Mandahl N, Mitelman F. Chromosome analysis of 96 uterine leiomyomas. Cancer Genet Cytogenet. 1991;55:11-18.

[2] Hennig Y, Rogalla P, Wanschura S, Frey G, Deichert U, Bartnitzke S, Bullerdiek J. HMGIC expressed in a uterine leiomy- oma with a deletion of the long arm of chromosome 7 along with a 12q14-15 rearrangement but not in tumors showing $\operatorname{del}(7)$ as the sole cytogenetic abnormality. Cancer Genet Cytogenet. 1997;96:129-133.

[ 3] Townsend DE, Sparkes RS, Baluda MC, McClelland G. Unicellular histogenesis of uterine leiomyomas as determined by electrophoresis by glucose-6-phosphate dehydrogenase. Am J Obstet Gynecol. 1970;107:1168-1173.

[4] Thornberry NA, Bull HG, Calaycay JR, Chapman KT, Howard AD, Kostura MJ, Miller DK, Molineaux SM, Weidner JR, Aunins J, Schmidt J.A., Tocci M. A novel heterodimeric cysteine protease is required for interleukin-1 beta processing in monocytes. Nature. 1992;356:768-774.

[ 5] Rothe M, Sarma V, Dixit VM, Goeddel DV. TRAF2-mediated activation of NF-kappa B by TNF receptor 2 and CD40. Science. 1995;269:1424-1427.

[ 6] Fotin-Mleczek M, Henkler F, Hausser A, Glauner H, Samel D, Graness A, Scheurich P, Mauri D, Wajant H. Tumor necrosis factor receptor-associated factor (TRAF) 1 regulates CD40-induced TRAF2-mediated NF-kappaB activation. $J$ Biol Chem. 2004;279:677-685.

[ 7] Ha H, Han D, Choi Y. TRAF-mediated TNFR-family signaling. Curr Protoc Immunol. 2009; Chapter 11:Unit11.9D.

[ 8] Hsu H, Shu HB, Pan MG, Goeddel DV. TRADD-TRAF2 and TRADD-FADD interactions define two distinct TNF receptor 1 signal transduction pathways. Cell. 1996;84:299-308.

[9] Cao Z, Xiong J, Takeuchi M, Kurama T, Goeddel DV. TRAF6 is a signal transducer for interleukin-1. Nature. 1996;383:443-446.

[10] Knop J, Wesche H, Lang D, Martin MU. Effects of overexpression of IL-1 receptor-associated kinase on NfkappaB activation, IL-2 production and stress-activated protein kinases in the murine T cell line EL4. Eur J Immunol. 1998;28:3100-3109.

[11] Li X, Commane M, Jiang Z, Stark GR. IL-1-induced NFkappa $\mathrm{B}$ and c-Jun N-terminal kinase (JNK) activation diverge at IL-1 receptor-associated kinase (IRAK). Proc Natl Acad Sci USA. 2001;98:4461-4465.

[12] Baud V, Liu ZG, Bennett B, Suzuki N, Xia Y, Karin M. Signaling by proinflammatory cytokines: oligomerization of TRAF2 and TRAF6 is sufficient for JNK and IKK activation and target gene induction via an amino-terminal effector domain. Genes Dev. 1999;13:1297-1308.

[13] Jobin C, Holt L, Bradham CA, Streetz K, Brenner DA, Sartor RB. TNF receptor-associated factor- 2 is involved in both IL1 beta and TNF-alpha signaling cascades leading to NF-kappa $\mathrm{B}$ activation and IL-8 expression in human intestinal epithelial cells. J Immunol. 1999;162:4447-4454.

[14] Wajant H, Scheurich P. Tumor necrosis factor receptor-associated factor (TRAF) 2 and its role in TNF signaling. Int $J$ Biochem Cell Biol. 2001;33:19-32.

[15] Malinin NL, Boldin MP, Kovalenko AV, Wallach D. MAP3Krelated kinase involved in NF-kappaB induction by TNF, CD95 and IL-1. Nature. 1997;385:540-544.

[16] Harper N, Hughes M, MacFarlane M, Cohen GM. Fas-associated death domain protein and caspase- 8 are not recruited to the tumor necrosis factor receptor 1 signaling complex during tumor necrosis factor-induced apoptosis. J Biol Chem. 2003;278:25534-25541.

[17] Awane M, Andres PG, Li DJ, Reinecker HC. NF-kappa Binducing kinase is a common mediator of IL-17-, TNF-alpha, and IL-1 beta-induced chemokine promoter activation in intestinal epithelial cells. J Immunol. 1999;162:5337-5344.

[18] Madej P, Plewka A, Plewka D, Paleń P, Nowaczyk G, Bogunia E, Marczyński J, Waloszek J. The aromatase expression in myomas and myometriums of women in reproduction and perimenopausal age. Folia Cytochem Cytobiol. 2009;47:497-504.

[19] Cramer SF, Patel A. The frequency of uterine leiomyomas. Am J Clin Pathol. 1990;94:435-438. 
[20] Houston KD, Hunter DS, Hodges LC, Walker CL. Uterine leiomyomas: mechanisms of tumorigenesis. Toxicol Pathol. 2001;29:100-104.

[21] Colgan TJ, Pron G, Mocarski EJ, Bennett JD, Asch MR, Common A. Pathologic features of uteri and leiomyomas following uterine artery embolization for leiomyomas. Am J Surg Pathol. 2003;27:167-177.

[22] Mizukami J, Takaesu G, Akatsuka H, Sakurai H, NinomiyaTsuji J, Matsumoto K, Sakurai N. Receptor activator of NFkappaB ligand (RANKL) activates TAK1 mitogen-activated protein kinase kinase kinase through a signaling complex containing RANK, TAB2, and TRAF6. Mol Cell Biol. 2002;22:992-1000.

[23] Takatsuna H, Kato H, Gohda J, Akiyama T, Moriya A, Okamoto Y, Yamagata Y, Otsuka M, Umezawa K, Semba K, Inoue J. Identification of TIFA as an adapter protein that links tumor necrosis factor receptor-associated factor 6 (TRAF6) to interleukin-1 (IL-1) receptor-associated kinase-1 (IRAK-1) in IL-1 receptor signaling. J Biol Chem. 2003;278:12144-12150.

[24] Yanagisawa K, Tago K, Hayakawa M, Ohki M, Iwahana H, Tominaga S. A novel splice variant of mouse interleukin-1receptor-associated kinase-1 (IRAK-1) activates nuclear factor-kappaB (NF-kappaB) and c-Jun N-terminal kinase (JNK). Biochem J. 2003;370:159-166.

[25] Song HY, Régnier CH, Kirschning CJ, Goeddel DV, Rothe M. Tumor necrosis factor (TNF)-mediated kinase cascades: bifurcation of nuclear factor-kappaB and c-jun N-terminal kinase (JNK/SAPK) pathways at TNF receptor-associated factor 2. Proc Natl Acad Sci USA. 1997;94:9792-9796.

[26] Darnay BG, Ni J, Moore PA, Aggarwal BB. Activation of NFkappaB by RANK requires tumor necrosis factor receptorassociated factor (TRAF) 6 and NF-kappaB-inducing kinase. Identification of a novel TRAF6 interaction motif. $J$ Biol Chem. 1999;274:7724-7731.

[27] Bradley JR, Pober JS. Tumor necrosis factor receptor-associated factors (TRAFs). Oncogene. 2001;20:6482-6491.

[28] Duckett CS, Thompson CB. CD30-dependent degradation of TRAF2: implications for negative regulation of TRAF signaling and the control of cell survival. Genes Dev. 1997; 11:2810-2821.

[29] Schwandner R, Yamaguchi K, Cao Z. Requirement of tumor necrosis factor receptor-associated factor (TRAF)6 in interleukin 17 signal transduction. J Exp Med. 2000;191:1233-1240.

[30] Arch RH, Gedrich RW, Thompson CB. Translocation of TRAF proteins regulates apoptotic threshold of cells. Biochem Biophys Res Commun. 2000;272:936-945.

[31] Schneider C, Hübinger G. Pleiotropic signal transduction mediated by human CD30: a member of the tumor necrosis factor receptor (TNFR) family. Leuk Lymphoma. 2002;43:1355-1366.

[32] Duckett CS, Thompson CB. The control and execution of programmed cell death: an update. Biochim Biophys Acta. 1997;1332:R454-457.

[33] Takaesu G, Ninomiya-Tsuji J, Kishida S, Li X, Stark GR, Matsumoto K. Interleukin-1 (IL-1) receptor-associated kinase leads to activation of TAK1 by inducing TAB2 translocation in the IL1 signaling pathway. Mol Cell Biol. 2001;21:2475-2484.

[34] Yeh WC, Shahinian A, Speiser D, Kraunus J, Billia F, Wakeham A, de la Pompa JL, Ferrick D, Hum B, Iscove N, Ohashi P, Rothe M, Goeddel DV, Mak TW. Early lethality, functional NF-kappaB activation, and increased sensitivity to TNFinduced cell death in TRAF2-deficient mice. Immunity. 1997:7:715-725.

[35] Wullaert A, Heyninck K, Beyaert R. Mechanisms of crosstalk between TNF-induced NF-kappaB and JNK activation in hepatocytes. Biochem Pharmacol. 2006;72:1090-1101.

[36] Hu P, Han Z, Couvillon AD, Kaufman RJ, Exton JH. Autocrine tumor necrosis factor alpha links endoplasmic reticulum stress to the membrane death receptor pathway through IRE1alpha-mediated NF-kappaB activation and down-regulation of TRAF2 expression. Mol Cell Biol. 2006;26:3071-3084.

Submitted: 15 February, 2010 Accepted after reviews: 19 May, 2010 